Electronic transport properties of Ti-impurity band in $\mathrm{Si}$

This content has been downloaded from IOPscience. Please scroll down to see the full text. 2009 J. Phys. D: Appl. Phys. 42085110

(http://iopscience.iop.org/0022-3727/42/8/085110)

View the table of contents for this issue, or go to the journal homepage for more

Download details:

IP Address: 147.96.14.15

This content was downloaded on 12/02/2014 at 16:34

Please note that terms and conditions apply. 


\title{
Electronic transport properties of Ti-impurity band in Si
}

\author{
J Olea, G González-Díaz, D Pastor and I Mártil
}

Departamento de Física Aplicada III (Electricidad y Electrónica), Facultad de Ciencias, Físicas, Universidad Complutense, E-28040 Madrid, Spain

Received 3 November 2008, in final form 3 February 2009

Published 1 April 2009

Online at stacks.iop.org/JPhysD/42/085110

\begin{abstract}
In this paper we show that pulsed laser melted high dose implantation of $\mathrm{Ti}$ in $\mathrm{Si}$, above the Mott transition, produces an impurity band (IB) in this semiconductor. Using the van der Pauw method and Hall effect measurements we find strong laminated conductivity at the implanted layer and a temperature dependent decoupling between the Ti implanted layer (TIL) and the substrate. The conduction mechanism from the TIL to the substrate shows blocking characteristics that could be well explained through IB theory.

Using the ATLAS code we can estimate the energetic position of the IB at $0.36 \mathrm{eV}$ from the conduction band, the density of holes in this band which is closely related to the Ti atomic density and the hole mobility in this band.

Band diagrams of the structure at low and high temperatures are also simulated in the ATLAS framework. The simulation obtained is fully coherent with experimental results.
\end{abstract}

\section{Introduction}

Conversion efficiency of single junction $\mathrm{Si}$ solar cells has reached, under non-concentrated illumination conditions, values in the range 24-26\% [1]. At the present moment, it seems a difficult task to improve these values optimizing the cell design or the fabrication procedure.

In 1997, a seminal paper was published in which a new concept, the so-called intermediate band (IB) [2], was introduced. According to this paper, single junction solar cells based on a semiconductor with an IB can reach efficiency values up to $63.2 \%$, for a host semiconductor with a band gap of $1.94 \mathrm{eV}$. This value is well above the maximum theoretical efficiency for single junction cells [3]. Whatever the material used to build up the device, the predicted efficiencies are well above those obtained today on multijunction, multilayer solar cells [4].

In recent years, theoretical studies have been conducted on a wide range of semiconductors as potential candidates in which the IB can be formed by means of the introduction in the host lattice of several transition elements [5]. Such studies demonstrate the formation of the IB by showing the density of states (DOS) distribution within the forbidden gap, in which a 'metallic band' (i.e. a semi-filled band) is obtained. More recently, a few papers have shown by means of optical characterization of different semiconductors such as GaInNAs
[6], ZnMnOTe [7] and GaNAsP [8] that an IB is present on such semiconductors. Additionally, the extension of the IB concept to quantum dot devices has been theoretically and experimentally proved [9].

Electrical transport properties of semiconductors with an IB could be, in principle, quite different from those of 'standard' semiconductors and, as a consequence, a careful analysis of such properties seems to be mandatory. In fact, in III-V and II-VI semiconductors such as GaN and $\mathrm{ZnO}$, an unintentionally introduced impurity band seems to be always present $[10,11]$. In the first semiconductor, a highly conductive layer that appears in the first stages of $\mathrm{GaN}$ growth [12] has been observed at the GaN/substrate interface. In the second case, a surface conductive layer has usually been reported on $\mathrm{ZnO}$ single crystals whatever the crystal growth procedure [13]. The influence of such conductive layers on the electrical transport properties of both semiconductors has been clarified by Hall effect measurements [14, 15]. Anyway the role of such conductive layers on device performance is a cause of concern $[12,13]$.

Regarding photovoltaic devices, the intentional introduction of an IB is considered a milestone on the road to achieving the goal of high efficiency devices [2]. In a recent paper, it is pointed out that the potential efficiency for single junction solar cells based on Si with such an IB would be very high [16]. This gives $\mathrm{Si}$ a place in the new generation of solar cells materials. 
In this paper we analyse the electrical transport properties of single crystal Si in which an IB is formed by means of two non-equilibrium techniques: Ti ion implantation at doses well above the Mott limit transition for this element [17] and subsequent pulsed laser melting (PLM) of the implanted layer to recover the lattice damage. This study is an experimental demonstration of the achievement of an IB in single crystal Si.

\section{Experimental}

Single crystal $\mathrm{n}-\mathrm{Si}$ samples $(\rho=200 \Omega \mathrm{cm} ; \mu=$ $1250 \mathrm{~cm}^{2} \mathrm{~V}^{-1} \mathrm{~s}^{-1} ; n=2.2 \times 10^{13} \mathrm{~cm}^{-3}$ measured at $300 \mathrm{~K}$ ) were implanted with Ti ions by means of a VARIAN CF3000 Ion Implanter refurbished by IBS. Ti was implanted at $30 \mathrm{keV}$, with three different doses: $10^{15}, 5 \times 10^{15}$ and $10^{16} \mathrm{~cm}^{-2}$. Then, the implanted Si samples were annealed by means of the PLM method [18] to activate the Ti impurities. The PLM annealing process was performed by J P Sercel Associates Inc. (New Hampshire, USA). Samples were annealed with one $20 \mathrm{~ns}$ long pulse of a $\mathrm{KrF}$ excimer laser $(248 \mathrm{~nm})$ at energy densities from 0.2 to $0.8 \mathrm{~J} \mathrm{~cm}^{-2}$. PLM is a highly non-equilibrium processing technique which is able to melt and recrystallize the Si surface up to about $100 \mathrm{~nm}$ deep in very short times $\left(10^{-8}-10^{-6} \mathrm{~s}\right)$. This rapid recrystallization time allows the incorporation of $\mathrm{Ti}$ atoms to $\mathrm{Si}$ at concentrations well above the solubility limit for this element [19]. Also, the PLM processing of the Ti implanted Si layer prevents secondary phase formation (i.e. titanium silicide) even when the equilibrium solubility limit has been greatly exceeded [20].

Time of flight secondary ion mass spectroscopy (ToF-SIMS) measurements were performed with a TOF_SIMS IV model manufactured by ION-TOF, using a $25 \mathrm{keV}$ pulsed $\mathrm{Ga}^{+}$beam at $45^{\circ}$ incidence and $\mathrm{O}_{2}$ flow. The raw profiles were calibrated by standard procedures, i.e. the concentration values were calculated from the ToF-SIMS counts by matching the Ti content in the implanted but not the annealed sample to the nominal amount of Ti and the depth was calculated by optical profilometry measurement of the crater depth and assuming a constant erosion rate.

Glancing incidence $\mathrm{x}$-ray diffraction (GIXRD) was performed to study the crystalline recovery of the implanted samples after the PLM process. A Philips X'pert Pro MRD diffractometer with $\mathrm{Cu} \mathrm{K}_{\alpha}$ radiation in glancing incidence configuration was used [21].

Channelling and random Rutherford backscattering spectroscopy (RBS) measurements were done to determine how the $\mathrm{Ti}$ atoms were incorporated into the $\mathrm{Si}$ lattice (i.e. substitutional or interstitial). Measurements were conducted in a Cockcroft-Walton accelerator built by High Voltage Energy Europe. The equipment worked with $2 \mathrm{MeV}$ He ions.

Finally, the $\mathrm{Si}$ implanted samples were electrically characterized by the van der Pauw method and Hall effect measurements at a variable temperature using a Keithley SCS 4200 model with four source and measure units. The samples were placed inside a homemade cryostat attached to a vacuum pump to avoid moisture condensation. The temperature was varied between 90 and $380 \mathrm{~K}$. We measured the four van

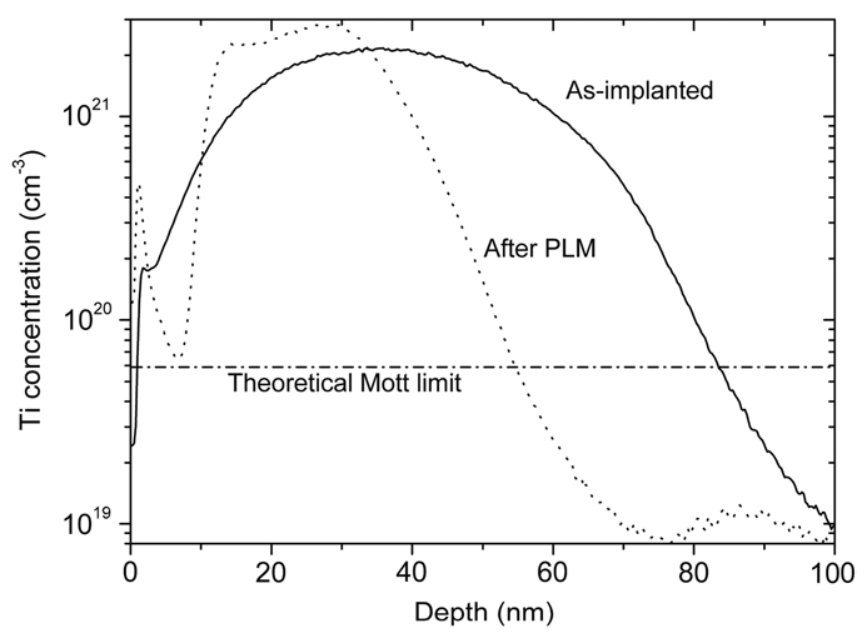

Figure 1. ToF-SIMS Ti concentration versus depth in the implanted layer. As-implanted sample (- - and annealed at $0.6 \mathrm{~J} \mathrm{~cm}^{-2}(---)$. Both samples have been implanted with the same dose of Ti atoms $\left(10^{16} \mathrm{~cm}^{-2}\right)$

der Pauw configurations and also changed the polarity of the measurement current source and the direction of the magnetic field to avoid any possibility of having spurious thermogalvanomagnetic effects.

\section{Results and discussion}

Figure 1 shows the ToF-SIMS profile of a sample implanted at the highest dose used $\left(10^{16} \mathrm{~cm}^{-2}\right)$, before and after the PLM process at $0.6 \mathrm{~J} \mathrm{~cm}^{-2}$, that is the minimum energy density that melts the Ti implanted layer (TIL). It can be seen how the implanted profile is slightly shifted to the Si surface after annealing. Profile integration gives us the percentage of the implanted $\mathrm{Ti}$ atoms remaining in the Si host lattice after annealing. Depending on PLM energy, that percentage ranges from $90 \%$ at $0.2 \mathrm{~J} \mathrm{~cm}^{-2}$ to $60 \%$ at $0.8 \mathrm{~J} \mathrm{~cm}^{-2}$. This means that the Ti atom concentration [Ti] that remains within the $\mathrm{Si}$ lattice after the PLM process was $1.2 \times 10^{20}, 6 \times 10^{20}$ and $12 \times 10^{20} \mathrm{~cm}^{-3}$ for $10^{15}, 5 \times 10^{15}$ and $10^{16} \mathrm{~cm}^{-2}$ doses, for $0.8 \mathrm{~J} \mathrm{~cm}^{-2} \mathrm{PLM}$ energy, assuming a constant profile of $50 \mathrm{~nm}$ depth. These values are well above the Mott transition limit for this element [17].

Concerning crystallinity of the TIL, GIXRD measurements showed that, after the PLM process, all TILs show a good degree of crystallinity recovery, being better for those samples implanted at the lowest dose and annealed with the highest energy. Samples implanted at the highest dose $\left(10^{16} \mathrm{~cm}^{-2}\right)$ do not show a complete lattice recovery after annealing even at the highest PLM energy, indicating a polycrystalline character of the TIL at these implantation doses. As a consequence, caution should be exercised with the obtained results on these samples [21].

RBS measurements showed that most of the Ti was located as interstitials (about $98-99 \%$ of the atoms remaining after annealing) [21].

As described elsewhere in the literature [22], Ti implanted atoms on $\mathrm{Si}$ are energetically located in centres at about $E_{\mathrm{C}}-0.3 \mathrm{eV}$, though some different centres have been reported, 




Figure 2. Schematic three-dimensional view. Van der Pauw set-up.

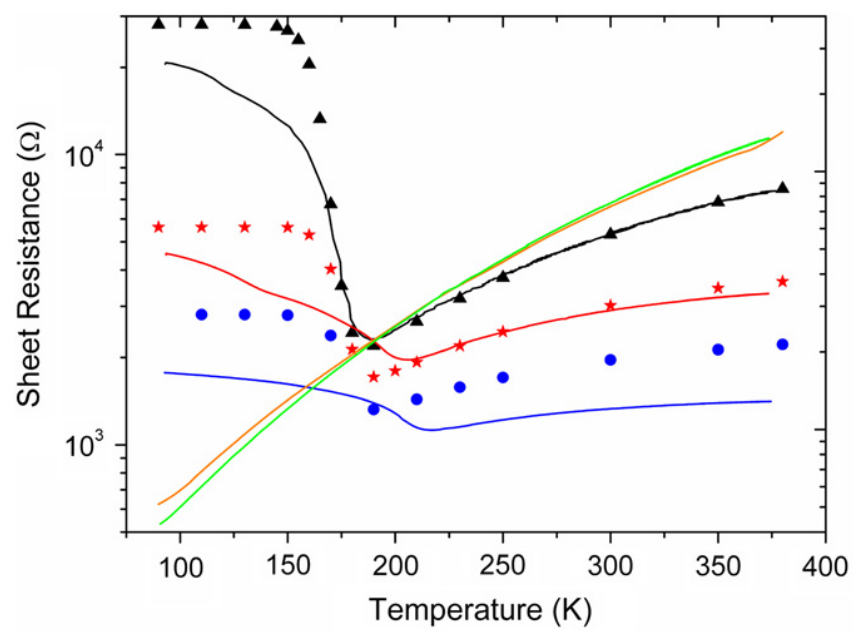

Figure 3. Sheet resistance as a function of the measured temperature for the $\mathrm{n}$-Si substrate (red line), for a PLM annealed $\mathrm{n}$-Si substrate (green line) and double sheet TIL/n-Si substrate for different implantation doses: $10^{15} \mathrm{~cm}^{-2}$ (black line: experimental;

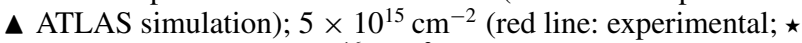
ATLAS simulation) and $10^{16} \mathrm{~cm}^{-2}$ (blue line: experimental; ATLAS simulation).

depending on the characterization technique used [23]. In any case, because in our samples the Ti concentration is above the Mott limit, overlapping of wavefunctions takes place and, as a consequence, the energetic location of the IB would be a rough estimation rather than a precise level.

A schematic drawing of the van der Pauw set-up with geometrical details is depicted in figure 2. From the figure, we have a double sheet (TIL/n-Si) and van der Pauw contacts are at the TIL surface corners.

\subsection{Sheet resistance}

Figure 3 shows the sheet resistance as a function of the measured temperature for an unimplanted unannealed $\mathrm{Si}$ substrate, an unimplanted annealed substrate and for the double sheet at the three implanted doses analysed (PLM energy: $0.8 \mathrm{~J} \mathrm{~cm}^{-2}$ in all cases). The sheet resistance of the annealed but not implanted Si substrates is plotted in the figure as a control test to show that there is no effect due just to PLM. For the implanted samples, an unexpected behaviour is observed



TIL

Si substrate

Figure 4. Generic band diagram of the TIL/n-Si double sheet. This drawing is not to scale and corresponds to the expected band diagram at $T=90 \mathrm{~K}$

for temperatures lower than $180-190 \mathrm{~K}$. The sheet resistance of the whole sample is higher than the sheet resistance of the substrate. This is odd if both the TIL and the substrate have the same majority carrier type, because the combined resistance of the two branches in parallel cannot exceed the resistance of any of them. This means that an electrical decoupling between the substrate and the TIL seems to take place for this temperature range. In this sense we will show that for these temperatures we are measuring only the transport properties of the TIL. For temperatures higher than 180-190 K, the measured sheet resistance is the expected parallel equivalent to the double sheet (TIL/n-Si substrate).

In the following, in order to explain the results, we will assume that an IB has been formed in the TIL. In fact, making some simple approximations, the critical impurity density at which the delocalization Mott transition takes place results in $N_{\text {Tcrit }}=5.9 \times 10^{19} \mathrm{~cm}^{-3}$ at $300 \mathrm{~K}$ [2]. The total Ti concentration is well above this number for all the implanted doses analysed here. As a consequence, delocalization of the impurity electron wavefunctions should take place and the IB is formed in the TIL.

According to IB theory, the band diagram of the TIL/n$\mathrm{Si}$ double sheet should be like the one shown in figure 4 . On the TIL side the Fermi level (FL) is pinned at the IB due to the high DOS at this energy and is almost constant with the temperature. Electrons at the conduction band (CB) and holes at the valence band (VB) are determined through standard Maxwell-Boltzmann statistics and its densities are strongly dependent on the temperature in spite of the constant position of the FL. Nevertheless, the carrier concentration at the IB should not agree with these statistics and they will behave like a degenerate band, in agreement with the theory for semiconductors with the IB [2]. The main concern now is the nature of the carriers in this band that depends on the band curvature and its filling degree. Now, the TIL behaves like an n-type or p-type semiconductor depending on the filling of IB and the corresponding effective mass of the carriers. Our experimental results show that this band is p-type as we will 
see later. According to the band diagram of figure 4, holes in the IB cannot cross to the substrate due to the height of the barrier from the TIL to the substrate VB. Therefore, at every temperature, holes are electrically confined in the TIL plane, with a low mobility corresponding to a narrow, high carrier density band.

At a low temperature, if the IB (and FL) is deep enough, the electron density should be very low at the TIL CB. In the substrate, the electron concentration is almost temperature independent (from 90 to $300 \mathrm{~K}$ ) corresponding to a semiconductor with an n-type shallow doping of $2.2 \times$ $10^{13} \mathrm{~cm}^{-3}$. The substrate FL position can be easily calculated using the Maxwell-Boltzmann statistics. At $90 \mathrm{~K}$ the FL in the substrate is $0.1 \mathrm{eV}$ below the CB. In figure 4 we have assumed that the IB is deeper than this energy [22]. The electrical connection between the two layers becomes unidirectional; the substrate can inject electrons to the TIL if the net applied voltage is enough so that the electrons could surmount the barrier energy but the TIL cannot inject electrons to the substrate because electron density at TIL is negligible at this temperature. According to figure 4, holes at the TIL VB or the substrate VB are negligible. In a parallel double sheet the fact that there is unidirectional contact between the layers means an effective decoupling of them because any current line coming to the substrate from the TIL and returning to the TIL from the substrate has to cross the junction twice in opposite directions.

As the temperature increases, the electron density in the TIL CB arises corresponding to the thermal generation from a level of $E_{\mathrm{C}}-E_{\mathrm{IB}}$ energy. This activation energy is smaller than the $\mathrm{Si}$ intrinsic one and density of electrons increases relatively fast. There should be a temperature at which the electron density in the TIL becomes similar to the substrate electron density. At this point, the barrier height for electrons should be very small or none. Electrons can now flow freely back and forth from one layer to another and both layers are electrically coupled. In addition to the position of the IB, there are some other parameters we have to estimate before we are able to calculate the barrier height between the TIL and the substrate.

1. DOS and hole concentration in the IB: as stated before, $\mathrm{Ti}$ is located at interstitial positions. As the Ti electronic configuration is $1 s^{2} 2 s^{2} 2 p^{6} 3 s^{2} 3 p^{6} 4 s^{2} 3 d^{2}$, each atom is able to give one to four electrons to the IB. If we assume as a first approach that each Ti atom gives an electron to the IB, the DOS should be $2 \times$ [Ti], in agreement with the standard semiconductor theory of band formation [24] and the hole density will be the Ti concentration [Ti], which are the empty states at the IB. Further in the paper we have some considerations about the possibility of having more electrons per Ti atom. Whanon and co-workers [25] have showed through ab initio calculations that the Ti interstitial atoms in a host Si lattice should produce an IB. Now the current conduction at this band could be carried by electrons or holes, depending solely on the exact curvature of the $E-k$ relation and the corresponding effective mass.

2. From the statistical point of view, the IB is supposed to be narrow enough to be considered as a single level.
3. It is worth noting that, for an energy band narrow enough, $N_{\mathrm{V}}$ (equivalent DOS) and DOS should be roughly the same. Hence, we will use for $N_{\mathrm{V}}$ the DOS quoted above, i.e. $N_{\mathrm{V}}=2 \times[\mathrm{Ti}] \mathrm{cm}^{-3}$.

Assuming the previous hypothesis, it is easy to calculate the densities of carriers using the classical semiconductor equations. Let us suppose that the IB is located $0.36 \mathrm{eV}$ below the CB. As we will see in the next paragraph, this is the value that best fits the experimental results and it is not temperature dependent, and it is worth saying that it is close to the Ti level referred to in the literature [22].

With this hypothesis, at a low temperature $(90 \mathrm{~K})$ the electrons' carrier density in the TIL should be very low and the barrier height ( $\Delta E$, see figure 4 ) with the substrate is $0.26 \mathrm{eV}$. Conversely at $300 \mathrm{~K}$ the electron density in the TIL increases up to values similar to the electron concentration in the substrate. In that case there is no appreciable barrier for electrons and they can flow freely in both directions, depending on the external voltage polarity.

To fully confirm the qualitative explanation of the previous paragraphs, we have performed some simulations using the ATLAS code framework [26]. Because in this code it is not possible to define a semiconductor with an IB, we have defined the TIL sheet like a 'new' semiconductor with the following characteristics:

- The CB is the same as $\mathrm{Si}$, having the same equivalent DOS, mobility, affinity and so on.

- The gap of this 'new' semiconductor is the $E_{\mathrm{C}}-E_{\mathrm{IB}}$ energy, avoiding the VB at the TIL.

- $N_{\mathrm{V}}=2 \times[\mathrm{Ti}]$ as previously explained, being the hole concentration, $p=[\mathrm{Ti}]$

- Hole mobility has to be guessed but it is supposed to have a very low value corresponding to a narrow energy band. This key hypothesis will be experimentally confirmed in the next section.

The proposed band diagram, resulting from the ATLAS code, is depicted in figure 5 for two temperatures, 90 and $300 \mathrm{~K}$. Note that the TIL 'normal' VB has disappeared in this model in relation to the model proposed in figure 4 because, as stated before, holes in both 'normal' VBs do not have any significant role in the model due to its low carrier density.

We have also simulated the structure of figure 2 with the ATLAS code, obtaining the sheet resistance of samples with three different implantation doses analysed in this paper, all of them with $0.8 \mathrm{~J} \mathrm{~cm}^{-2}$ annealing. A comparison between the sheet resistance experimental data and the ATLAS simulation values is presented in figure 3 . In the fitting, only two parameters have been scanned: the IB energetic position and the hole mobility. The best fit is obtained with $E_{\mathrm{C}}-E_{\mathrm{IB}}=$ $0.36 \mathrm{eV}$ and hole mobility $\mu_{\mathrm{p}}=0.4 \mathrm{~cm}^{2} \mathrm{~V}^{-1} \mathrm{~s}^{-1}$. No fit is done either in the hole concentration which remains equal to the Ti concentration or in the $N_{\mathrm{V}}$ value at the IB that is $2 \times$ [Ti]. The sheet resistance minimum is very sensitive to the pseudo-gap chosen, i.e. the energetic position of the IB, being the constant value of the sheet resistance at low temperatures very dependent on the hole mobility times the IB hole density. 


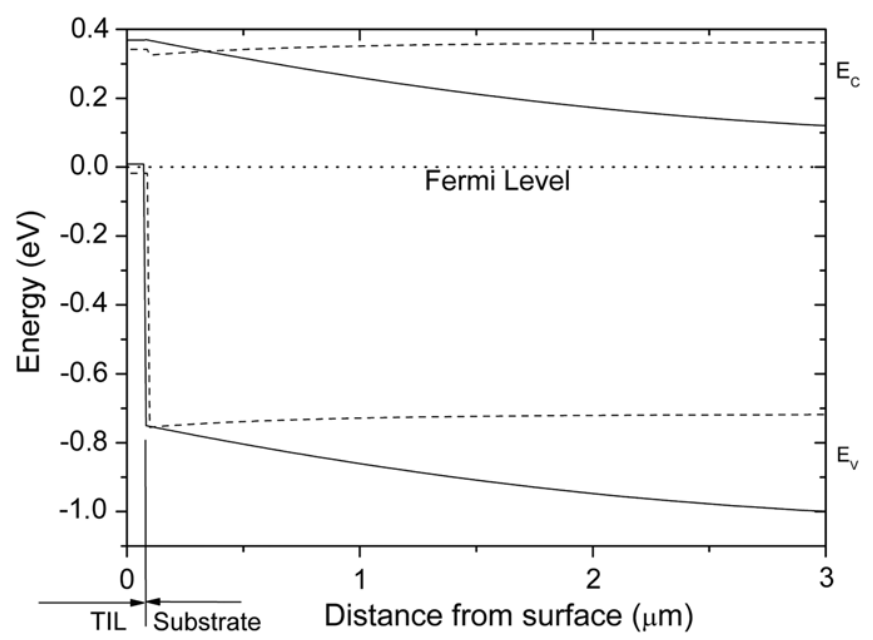

Figure 5. Band diagram, obtained from ATLAS simulation, of the double sheet quoted in figures 2 and 3 at $T=90 \mathrm{~K}\left(-E_{\mathrm{C}}\right.$ and $\left.-E_{\mathrm{V}}\right)$ and $T=300 \mathrm{~K}\left(---E_{\mathrm{C}}\right.$ and ---- $\left.E_{\mathrm{V}}\right)$.

For this reason we cannot know exactly how many carriers are given for each $\mathrm{Ti}$ atom; it goes from one electron per Ti atom with $0.4 \mathrm{~cm}^{2} \mathrm{~V}^{-1} \mathrm{~s}^{-1}$ mobility for the corresponding hole to four electrons with $0.1 \mathrm{~cm}^{2} \mathrm{~V}^{-1} \mathrm{~s}^{-1}$ mobility.

At high temperatures the fit to the sample implanted with the lowest dose $\left(10^{15} \mathrm{~cm}^{-2}\right)$ is excellent. For the $5 \times 10^{15} \mathrm{~cm}^{-2}$ sample, the fit is very good, while for the higher dose $\left(10^{16} \mathrm{~cm}^{-2}\right)$ there is a clear difference between the simulation and the experimental results. The more plausible explanation is that for high doses the crystallinity recovery is not perfect as the GIXRD measurements show clearly [21].

When the temperature decreases, the simulated sheet resistance increases faster than the experimental results. Additionally, the experimental results do not show a plateau as the simulation results do. Both differences could be related to a possible uncontrolled current leak through the TIL-substrate interface, mainly at the sample rim. As this is similar to the behaviour of a reverse polarized $\mathrm{p}-\mathrm{n}$ junction without special technology to avoid surface leaks, we suppose that here the most probable region to have superficial states is the rim of the sample which is a highly damaged zone. As a consequence, the path current through the rim would take place. This extra conduction path has not been simulated by the ATLAS code.

Figures $6(a)$ and $(b)$ show the simulated equipotential lines in a cross section from electrode 1 to electrode 2 of figure 2 at 90 and $300 \mathrm{~K}$. At high temperatures (figure 6(b)) the equal potential lines are perpendicular to the surface and there is no difference between the TIL and the substrate, meaning that the current is carried by both layers. At low temperatures the whole substrate has almost the potential of the positive electrode. As we justified before, the junction between the TIL and the substrate is blocking if TIL is negative and not blocking with the opposite polarity. This reasoning explains why the external potential drops between the substrate and the TIL at the negative electrode, being the substrate at the potential of the positive one.

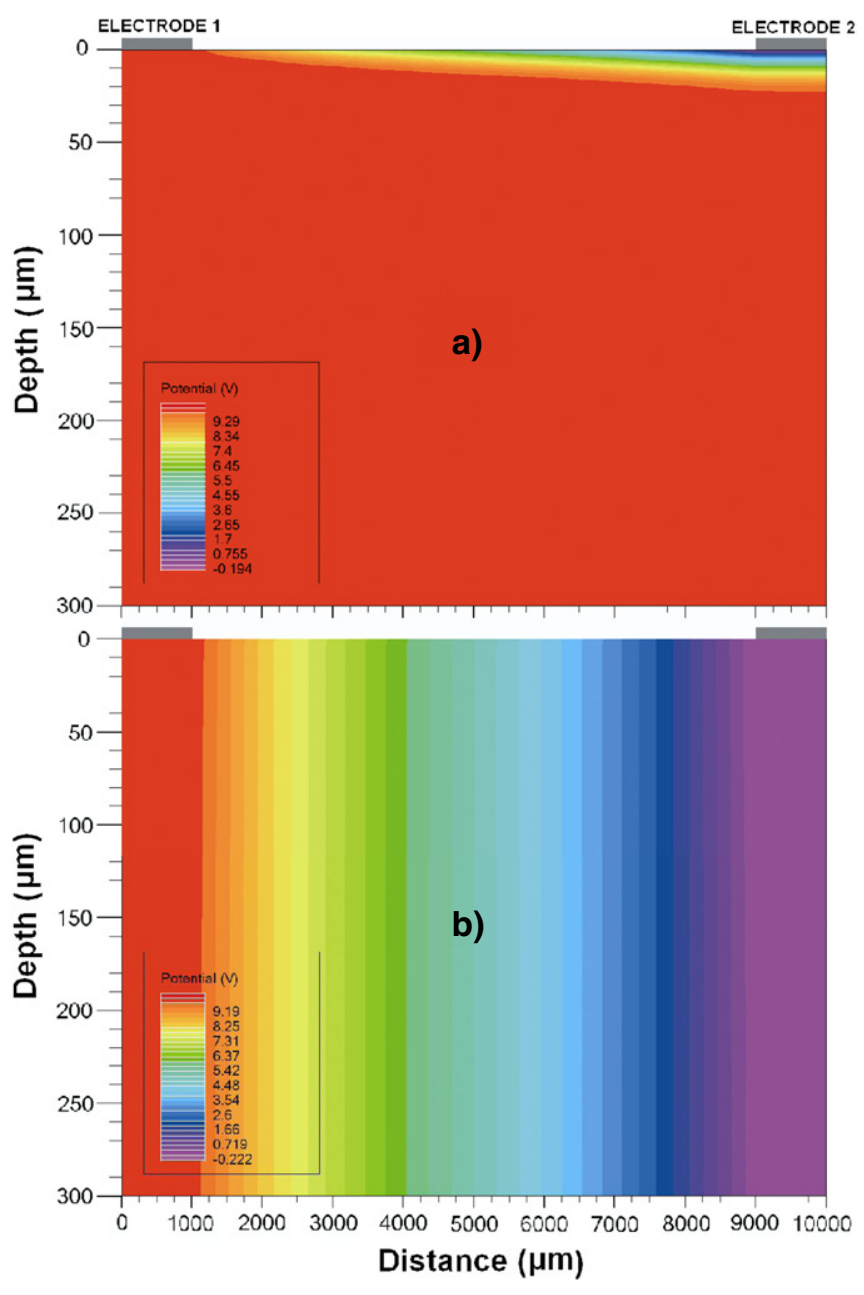

Figure 6. Potential distribution following the ATLAS code for $(a)$ $90 \mathrm{~K}$ and $(b) 300 \mathrm{~K}$

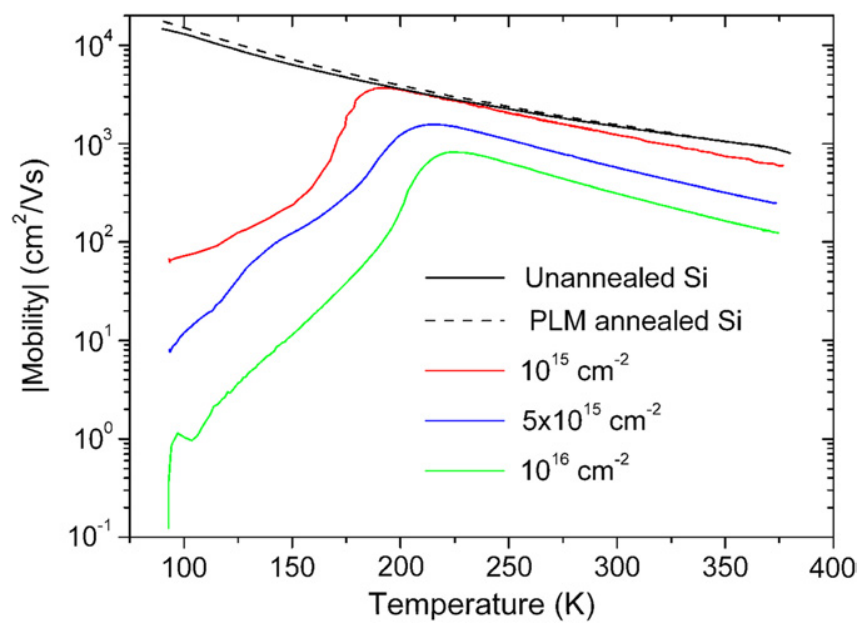

Figure 7. Hall mobility for the measured substrate (unannealed and PLM annealed) and double sheet as in figure $3: 10^{15} \mathrm{~cm}^{-2}$, $5 \times 10^{15} \mathrm{~cm}^{-2}$ and $10^{16} \mathrm{~cm}^{-2}$.

\subsection{Hall mobility}

Figure 7 shows a plot of the absolute value of the Hall mobility of the same samples measured in figure 3. The Hall mobility is negative, corresponding to an n-type semiconductor. While 
virgin and annealed substrates show the expected behaviour for the temperature dependence of the Hall mobility in a low doped Si single crystal (with the phonon scattering being present), this dependence for the implanted samples again shows an anomalous trend for temperatures lower than 180-190 K, i.e. for the same temperature range as in figure 3. For temperatures higher than $180 \mathrm{~K}$, the behaviour follows the same trend as the single crystal Si.

A qualitative explanation of the results in figure 7 could be obtained if we take into account that the mobility of the double sheet at high temperatures could be written as

$\mu_{\mathrm{H}}=\frac{\left(t_{1} \mu_{\mathrm{pl}}^{2} p_{1}+t_{\mathrm{s}} \mu_{\mathrm{ps}}^{2} p_{\mathrm{s}}+t_{\mathrm{l}} \mu_{\mathrm{IB}}^{2} p_{\mathrm{IB}}-t_{\mathrm{l}} \mu_{\mathrm{nl}}^{2} n_{\mathrm{l}}-t_{\mathrm{s}} \mu_{\mathrm{ns}}^{2} n_{\mathrm{s}}\right)}{\left(t_{1} \mu_{\mathrm{pl}} p_{1}+t_{\mathrm{s}} \mu_{\mathrm{ps}} p_{\mathrm{s}}+t_{\mathrm{l}} \mu_{\mathrm{IB}} p_{\mathrm{IB}}+t_{1} \mu_{\mathrm{nl}} n_{1}+t_{\mathrm{s}} \mu_{\mathrm{ns}} n_{\mathrm{s}}\right)}$,

where $t_{1}$ and $t_{\mathrm{s}}$ are the thicknesses of the layer and the substrate, respectively, $p_{1}$ is the TIL density of the holes in the VB, with $\mu_{\mathrm{pl}}$ its mobility, $p_{\mathrm{s}}$ and $\mu_{\mathrm{ps}}$ are analogous for the Si substrate, $p_{\mathrm{IB}}$ and $\mu_{\mathrm{IB}}$ are the hole density and mobility in the IB and $n_{1}$ and $\mu_{\mathrm{nl}}$ are the electron density and mobility in the TIL CB, with $n_{\mathrm{s}}$ and $\mu_{\mathrm{ns}}$ analogous as before for the substrate.

Assuming that the hole densities in the VB both in the TIL and in the substrate are negligible, the previous formula can be rewritten as

$$
\mu_{\mathrm{H}}=\frac{\left(t_{1} \mu_{\mathrm{IB}}^{2} p_{\mathrm{IB}}-t_{1} \mu_{\mathrm{nl}}^{2} n_{1}-t_{\mathrm{s}} \mu_{\mathrm{ns}}^{2} n_{\mathrm{s}}\right)}{\left(t_{1} \mu_{\mathrm{IB}} p_{\mathrm{IB}}+t_{1} \mu_{\mathrm{nl}} n_{1}+t_{\mathrm{s}} \mu_{\mathrm{ns}} n_{\mathrm{s}}\right)} .
$$

At high temperatures the terms corresponding to electrons of the equations should be higher than the hole term. The mobility is lower than in the $\mathrm{Si}$ substrate because of the influence of the hole term and also because the electrons in the TIL have a lower mobility than in the substrate. At temperatures lower than the critical one, the absolute value of the mobility decays due to the decrease in the electron concentration in the TIL and by the decoupling of the two sheets. The mobility drops faster for more doped samples.

As stated before, we have assumed throughout this paper that the main charge carriers in the IB are holes. There is further experimental evidence assessing the p-type character. In fact, in some samples (not shown in figure 3 or figure 7 for the sake of clarity), the positive Hall mobility was measured at low temperatures. Figure 8(a) shows the sheet resistance of a representative sample and figure $8(b)$ the Hall mobility in which p-type conduction has been measured. As can be seen in the inset of figure $8(b)$, the Hall mobility became positive for temperatures lower than $190 \mathrm{~K}$, i.e. for the temperatures where the anomalous behaviour of both the sheet resistance and the Hall mobility occurs. The low mobility value and the independence with the temperature are typical of charge carriers within a very narrow band [10]. The low mobility value is close to the $0.4 \mathrm{~cm}^{2} \mathrm{~V}^{-1} \mathrm{~s}^{-1}$ simulated value, temperature independent. Further evidence comes from the transversal measurements of which we have some preliminary results represented in figure 9 . These results are obtained with a guard ring, to avoid superficial leakages, and $\mathrm{n}^{+}$implantation in the back surface to set up an ohmic contact. Contacts to the TIL top layer were made by means of e-beam Al evaporated electrodes, without $\mathrm{a}^{+}$additional implanted layer due to the tiny thickness of the TIL (see figure 1, about $50 \mathrm{~nm}$ ). We have confidence in

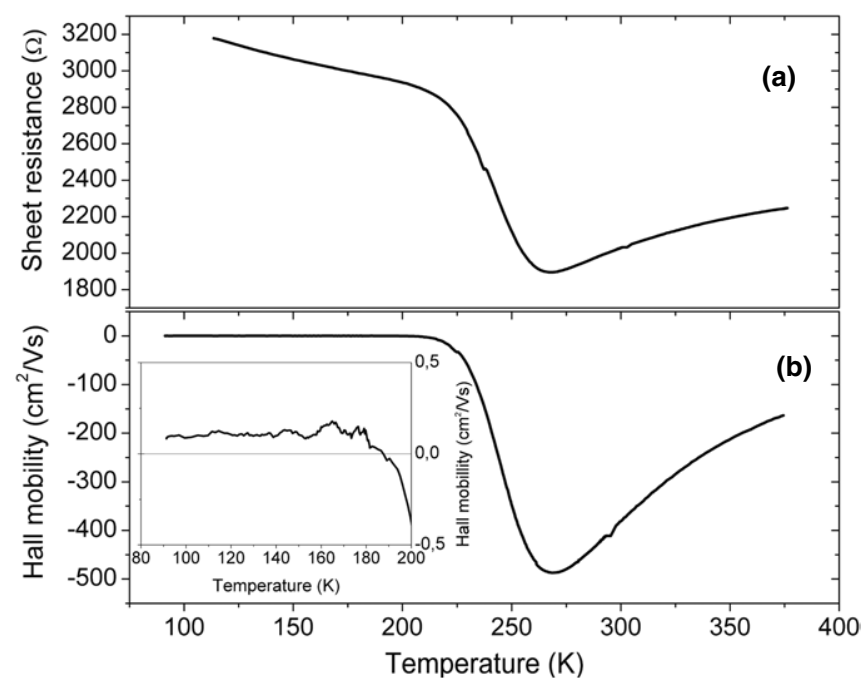

Figure 8. Sheet resistance $(a)$ and Hall mobility $(b)$ for a representative sample in which p-type conduction has been measured $\left(5 \times 10^{15} \mathrm{~cm}^{-2}\right.$ implantation dose; PLM at $\left.0.6 \mathrm{~J} \mathrm{~cm}^{-2}\right)$. The inset shows the Hall mobility in the low temperature zone.

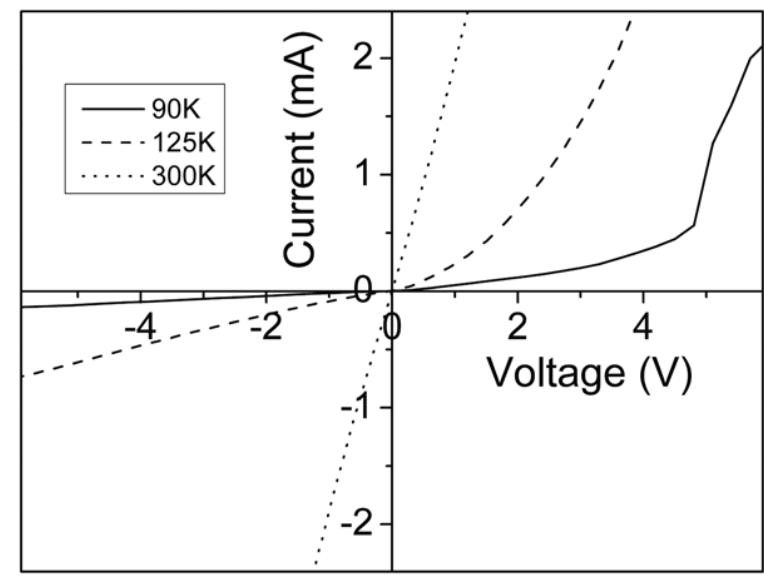

Figure 9. Transversal $I-V$ characteristics of the sample with an implantation dose of $10^{15} \mathrm{~cm}^{-2}$ and annealed with $0.8 \mathrm{~J} \mathrm{~cm}^{-2}$ measured at different temperatures. Note that at low temperatures a rectifying behaviour is observed while the ohmic one holds at a high temperature.

the ohmic nature of the TIL contact because the implanted layer has an extremely high carrier concentration. There is a clear rectification effect at low temperatures while the behaviour at room temperature is purely linear. This behaviour is fully congruent with the decoupling model.

\section{Conclusions}

In this paper we have shown that it is possible to build an IB in Si through the use of high dose $\left(10^{15}, 5 \times 10^{15}\right.$ and $10^{16} \mathrm{~cm}^{-2}$ ), low energy (30 keV) ion implantation of $\mathrm{Ti}$ and subsequent PLM. The transport properties of the implanted layers can be only explained assuming the existence of a p-type IB. Holes remain confined in the implanted layer giving a strong laminar conductivity. This layer remains isolated from the substrate at low temperature and connected to it above a critical temperature. The coupling/decoupling is associated 
with the electron density in the TIL and its rapid temperature variations.

Using the ATLAS code framework we were able to determine that the IB is located about $0.36 \mathrm{eV}$ below the CB. Also the hole mobility at the IB should be very small, as low as $0.4 \mathrm{~cm}^{2} \mathrm{~V}^{-1} \mathrm{~s}^{-1}$.

From the point of view of the ATLAS code, the TIL layer behaves like a degenerate p-type semiconductor with a $0.36 \mathrm{eV}$ gap, the CB identical to the $\mathrm{Si}$ and VB with an equivalent DOS and doping fully determined by the Ti concentration.

The results shown here are experimental evidence about how the IB influences the transport properties, which have been explained with non-conventional transport theory.

This result leads to the possibility of obtaining $\mathrm{Si}$ solar cells with the IB. This may also allow one think about a new generation of radiation detectors based on $\mathrm{Si}$, with the advantage of great technological maturity of such semiconductors.

\section{Acknowledgments}

The authors would like to acknowledge the Nanotechnology and Surface Analysis Services of the Universidad de Vigo C.A.C.T.I. for SIMS measurements, the Center for Microanalysis of Materials of the Universidad Autónoma de Madrid for RBS measurements, C.A.I. de difracción de rayos $\mathrm{X}$ of the Universidad Complutense de Madrid for GIXRD measurements and C.A.I. de Técnicas Físicas of the Universidad Complutense de Madrid for ion implantation experiments. This work was made possible thanks to the FPI (Grant No BES-2005-7063) of the Spanish Ministry of Education and Science. This work was partially supported by the Projects NUMANCIA (No S-0505/ENE/000310) founded by the Comunidad de Madrid and GENESIS-FV (No CSD2006-00004) founded by the Spanish Consolider National Programme and by U.C.M.-C.A.M. under Grant CCG07UCM/TIC-2804.

\section{References}

[1] Green M A, Emery K, Hisikawa Y and Warta W 2007 Prog. Photovolt.: Res. Appl. 15 425-30

[2] Luque A and Martí A 1997 Phys. Rev. Lett. 78 5014-7

[3] Shockley W and Queisser H J 1961 J. Appl. Phys. 32 510-9
[4] King R R, Law D C, Edmondson K M, Fetzer C M, Kinsey G S, Yoon H, Sherif R A and Karam N H 2007 Appl. Phys. Lett. 90183516

[5] Wahnón P and Tablero C 2002 Phys. Rev. B 65165115

[6] Shan W, Walukiewicz W, Ager III J W , Haller E E, Geisz J F, Friedman D J, Olson J M and Kurtz S R 1999 Phys. Rev. Lett. 82 1221-4

[7] Yu K M, Walukiewicz W, Wu J, Shan W, Beeman J W, Scarpulla M A, Dubon O D and Becla P 2003 Phys. Rev. Lett. 91246403

[8] Yu K M, Walukiewicz W, Ager III J W, Bour D, Farshchi R, Dubon O D, Li S X, Sharp D and Haller E E 2006 Appl. Phys. Lett. 88092110

[9] Luque A, Martí A, López N, Antolín E, Cánovas E, Stanley C, Farmer C, Caballero L J, Cuadra L and Balenzategui J L 2005 Appl. Phys. Lett. 87083505

[10] Hsu J W P, Lang D V, Richter S, Kleiman R N, Sergent A M, Look D C and Molnar R J 2001 J. Electron. Mater. 30 115-22

[11] Look D C 2007 Superlatt. Microstruct. 42 284-9

[12] Mavroidis C, Harris J J, Jackman R B, Bougrioua Z and Moerman I 2003 Diamond Relat. Mater. 12 1127-32

[13] Look D C, Chaflin B and Smith H E 2008 Appl. Phys. Lett. 92122108

[14] Look D C, Reynolds D C, Hemsky J W, Sizelove J R, Jones R L and Molnar R J 1997 Phys. Rev. Lett. 79 2273-6

[15] Look D C 2007 Surf. Sci. 601 5315-9

[16] Ley M, Boudaden J and Kuznicki Z T 2005 J. Appl. Phys. 98044905

[17] Mott N F 1968 Rev. Mod. Phys. 40 677-83

[18] Clark M H and Jones K S 2005 J. Appl. Phys. 97093525

[19] Yu K M, Walukiewicz W, Scarpulla M A, Dubon O D, Wu J, Jasinski J, Liliental-Weber Z, Beeman J W, Pillai M R and Aziz M J 2003 J. Appl. Phys. 94 1043-9

[20] White C W, Wilson S R, Appleton B R and Young F W Jr 1980 J. Appl. Phys. 51 738-49

[21] Olea J, Toledano-Luque M, Pastor D, González-Díaz G and Mártil I 2008 J. Appl. Phys. 104016105

[22] Mathiot D and Hocine S 1989 J. Appl. Phys. 66 5862-7

[23] Beeler F, Andersen O K and Scheffler M 1990 Phys. Rev. B 41 1603-24

[24] Conwell E M 1956 Phys. Rev. 103 51-61

[25] Sánchez K, Aguilera I, Palacios P and Wahnon P 2009 Assessment through first-principles calculations of a novel intermediate band photovoltaic material based on Ti-implanted silicon: interstitial versus substitutional origin Phys. Rev. A submitted

[26] ATLAS Device Simulator Framework distributed by Silvaco Data Syatems Inc. 4701 (Patrick Henry Drive Bldg\#6, Santa Clara, CA 95054) 